\title{
Ring-Type Magnitude Modulation for LINC: A Pragmatic Approach to the Efficiency Challenge
}

\author{
António Simões, Mário Castanheira, Marco Gomes, Member, IEEE, \\ Rui Dinis, Senior Member, IEEE, and Vitor Silva
}

\begin{abstract}
This paper considers the use of the linear amplification with nonlinear components (LINC) technique for the power amplification of spectrally compact offset quadrature phase shift keying (OQPSK) signals allowing the use of highly efficient, low cost, and strongly nonlinear high power amplifiers (HPAs). However, the performance of the LINC signal separation and power combining procedures decreases with the rise of the signal's peak-to-average power ratio (PAPR). A new ring-type magnitude modulation (RMM) method is proposed for OQPSK signals that limits both its maximum and minimum complex envelope excursions avoiding zero crossings, without spreading the transmitted signal's spectrum. The performance results show that band-limited OQPSK signals whose envelope has low fluctuations produce LINC components with a narrower spectrum, with a considerable impact on the LINC transmitter regardless of the type of combiner chosen: when using a passive/matched combiner, the transmitter's power efficiency is significantly increased without spreading the combined signal's spectrum; for the highly efficient non-linear Chireix combiner, there is a reduction of the amount of spectral leakage produced by nonlinearly combining the LINC signal components. Finally, an iterative decoding scheme is also proposed, which employs estimates of the received symbols' RMM coefficients to compensate the RMM distortion.
\end{abstract}

Index Terms-LINC transmitter, magnitude modulation, OQPSK signals, power efficiency.

\section{INTRODUCTION}

$\mathbf{T}$ HE USE of the millimeter wave (mm-wave) bands is expected to be a key feature of $5 \mathrm{G}$ (Fifth Generation) systems, since the large bands available allow significant capacity gains [1]-[3]. Moreover, the small wavelengths imply the use of small antennas that can be packed together with small separation between each other, allowing the development of massive multiple-input and multiple-output (MIMO) schemes. However, the deployment of mm-wave systems faces

Manuscript received August 2, 2016; revised February 2, 2017; accepted April 10, 2017. Date of publication April 18, 2017; date of current version August 14, 2017. This work was funded by FCT/MEC through national funds and when applicable co-funded by FEDER-PT2020 partnership agreement under the project UID/EEA/50008/2013. This work was published in the IEEE GLOBECOM'2015 [19], IEEE VTC'2016-Spring [20], and IEEE ICC'2016 [21]. The associate editor coordinating the review of this paper and approving it for publication was A. Ghrayeb. (Corresponding author: Marco Gomes.)

A. Simões was with the Instituto de Telecomunicações and the Department of Electrical and Computer Engineering, University of Coimbra, 3000-213 Coimbra, Portugal. He is now with Instituto Superior Técnico, Technical University of Lisbon, 1049-001 Lisbon, Portugal

M. Castanheira, M. Gomes, and V. Silva are with the Instituto de Telecomunicações and the Department of Electrical and Computer Engineering, University of Coimbra, 3000-213 Coimbra, Portugal.

R. Dinis is with Instituto de Telecomunicações and DEE-FCT, Universidade Nova of Lisboa, 2829-516 Caparica, Portugal.

Color versions of one or more of the figures in this paper are available online at http://ieeexplore.ieee.org.

Digital Object Identifier 10.1109/TCOMM.2017.2695602 important challenges regarding the channel propagation constraints (this is characterized by high free space path losses, small diffraction effects, and huge losses due to obstacles) and implementation difficulties at the hardware level. These issues combined with the large bit rates means that 5G's power requirements are much more severe than with conventional sub-6GHz communications, thus making efficient power amplification crucial for mm-wave communications.

The power amplification stage is in fact one of the critical components in the design of wireless transmitters. Most spectral efficient transmission techniques impose stringent linearity requirements with a consequent negative impact on power efficiency and HPA's cost; class A, AB or quasi-linear HPAs have to be used. In fact, the high PAPR of highorder constellation signals (e.g $m$-ary quadrature amplitude modulation (QAM)) add an additional efficiency penalty, since they require a larger back-off from the linear HPA's saturation point. These constraints have motivated the development of envelope controlling and PAPR reduction techniques over the last decades [4]-[7]. However, most of these techniques increase the transmitter's complexity, while not precluding the need for using linear or quasi linear amplifiers. Therefore, the strict power efficiency requirements of upcoming 5G systems, especially those operating at $\mathrm{mm}$-wave frequencies, crave for a different approach.

The LINC technique [8]-[10] has the potential to fill in that role, since it allows to perform linear amplification through the use of strongly nonlinear amplifiers such as class D or E amplifiers; these amplifiers have a much higher efficiency and a lower complexity, being much cheaper than linear or quasi linear HPAs [7], [11]. The LINC method consists in separating an input signal in two constant-envelope branches to be amplified separately by two highly efficient strongly non-linear amplifiers. Then, the two amplified components are combined in order to produce an output signal corresponding to the linear amplification of the input. In practice, the LINC signal components can be recombined using either: a passive combiner [9] which provides perfect linear signal combination at the expense of some LINC's overall power efficiency loss due to lower efficiency of the combiner; or a nonlinear Chireix combiner [12], highly efficient, though at the cost of a nonperfect linear combination of LINC's signal components.

While the LINC technique has a tremendous upside, its true potential is limited by the envelope characteristics of the input signal [9]. When LINC is used the amplitude information of the bandlimited signal to be amplified is carried in the LINC signal components' phase. A highly fluctuating envelope produces constant envelope LINC signal components with a high 
phase variation, thus having a much broader spectrum than the signal from which they were derived, resulting in an increase of the bandwidth that each nonlinear HPA needs to accommodate. Furthermore, it is also well known that for a LINC system employing a passive combiner the power efficiency tends to decrease as the signal's PAPR increases [9]. Both these LINC limitations can be addressed and mitigated by reducing the amplitude dynamic range of the signal to be amplified.

The use of modulations producing signals with low envelope fluctuations, such as OQPSK-type modulations, are therefore desirable for LINC; nevertheless, these are not usually regarded as good candidates to meet the spectral efficiency and high bit rates requirements of modern communication systems. Spectrally efficient transmission techniques manage to produce signals with compact spectrum (e.g., through filtering using a square-root raised-cosine filter with small roll-off), with the resulting signals having high envelope fluctuations and PAPR. The situation is significantly aggravated when large constellations (e.g., 16-QAM or 64-QAM constellations) are employed to further improve the system's spectral efficiency, and the resulting high PAPR signals may seem to limit the potential of LINC use. However, any M-ary constellation can be decomposed as a sum of several polar components [13], which can be modulated as OQPSK signals (also known as staggered QPSK [14]) and amplified and transmitted separately, eventually employing multi-layer massive antenna structures [15], [16] envisioned for 5G, enabling very efficient signal transmission provided that an efficient amplification for OQPSK signals with compact spectrum is employed.

The time offset between in-phase and quadrature components of OQPSK signals naturally results in lower envelope fluctuations and zero-crossing avoidance than with non-offset modulations. Nonetheless, OQPSK bandlimited signals close to the Nyquist limit still present substantial envelope fluctuations. Regarding this, magnitude modulation (MM) techniques [17], [18] are known to be effective methods of controlling the signal's envelope excursions without spreading the transmitted signal's spectrum or noticeably affecting transmission's bit error rate (BER) performance. However, when applied to bandlimited OQPSK signals, conventional magnitude modulation (MM) techniques only control the maximum upper envelope excursion, with the resulting signals still having a high dynamic range (although with lower PAPR), with frequent zero crossings, not allowing an efficient LINC transmission. This paper develops the recent proposed RMM method [19]-[21], especially designed for narrowband OQPSK signals, to be included in a single-carrier (SC) transmitter with a LINC power amplification stage (which for simplicity will be referred to hereafter as a LINC transmitter). Unlike conventional MM techniques, the RMM technique leads to OQPSK signals with compact spectrum and whose envelope has a low dynamic range and very low PAPR, leveraging an efficient power amplification. The performance of OQPSK when employing RMM is studied analytically for the additive white gaussian noise (AWGN) channel, and an efficient iterative receiver is proposed. Also, an overall study is made when combining RMM-OQPSK signals with LINC amplification considering both types of LINC combiners, i.e. passive and Chireix; different perspectives are considered and analyzed as a whole, namely, achievable efficiency gains, bandwidth requirements of nonlinear HPAs, robustness against HPAs' phase and gain imbalances and clipping. It is shown that combining the proposed RMM method with LINC is beneficial for both the passive and Chireix combiners.

This paper is organized as follows. Section II presents the LINC power amplification stage, along with the problems that restrain its implementation. In order to overcome these limitations, section III introduces the RMM method [19], [20]. After explaining its main features and the algorithm for computing the RMM coefficients, section IV presents the proposed transceiver scheme that combines RMM with the LINC technique, where it is explained the procedure to choose the RMM amplitude boundaries that address the LINC's challenges. In this section it is also presented an iterative decoding scheme that makes up for the distortion introduced by the RMM coefficients [21]. Section V explores the possible power efficiency and bandwidth tradeoffs that result from either using post-filtering clipping together with passive combiners or by employing a Chireix combiner instead. Concluding remarks are provided in section VI.

\section{LINC SYSTEMS}

The linear input-output power relationship requirement of various communication systems severely restricts the choice of the HPAs to be included in these systems, since linear HPAs have a substantially lower power efficiency than their nonlinear counterparts [7], [11]. The LINC technique [8]-[10] was developed to overcome these restraints, managing to achieve linear power amplification while employing highly efficient and grossly non-linear HPAs.

\section{A. Basic Concepts}

Generically, the LINC technique can be described by the following set of equations [8], [9]:

$$
\begin{aligned}
s(t) & =r(t) e^{j \phi(t)}=s_{1}(t)+s_{2}(t), \\
r(t) & =r_{\max } \cos (\theta(t)), \\
s_{i}(t) & =\frac{r_{\max }}{2} e^{j\left(\phi(t)+(-1)^{i} \theta(t)\right)} \text { for } i \in\{1,2\},
\end{aligned}
$$

where $s(t)$ is the signal to amplify with instantaneous amplitude $r(t) \geq 0$ and phase $\phi(t)$ (where both $r(t)$ and $\phi(t)$ are time-varying functions), which is decomposed as the sum of two constant envelope signals $s_{1}(t)$ and $s_{2}(t)$ that are separately amplified by highly efficient NL HPAs. The LINC's components, $s_{1}(t)$ and $s_{2}(t)$, are phase modulated signals with amplitude $r_{\max } / 2$ (where $r_{\max }=\max r(t)$ ) and instantaneous phase $\phi(t) \pm \theta(t)$, where $\theta(t)$ is the LINC branches' decomposition angle. Alternatively, (3) can be written as:

$$
s_{i}(t)=\frac{1}{2} s(t)\left(1+j(-1)^{i} e(t)\right) \text { for } i \in\{1,2\},
$$

where

$$
e(t)=\sqrt{\left(\frac{r_{\max }}{r(t)}\right)^{2}-1}
$$

is the scaling factor for the component in quadrature with the bandlimited signal $s(t)$. 


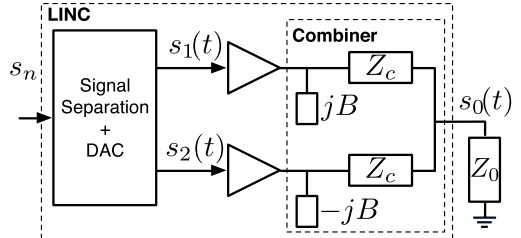

Fig. 1. Digital LINC power amplification stage, with a Chireix combiner.

Fig. 1 presents the structure of a LINC amplifier. While the LINC components $s_{1}(t)$ and $s_{2}(t)$ need to be linearly recombined to obtain an amplified replica of the input signal $s(t)$ (typically using a passive/matched combiner), this combining method offers low efficiency when dealing with signals with high envelope fluctuations [9]. Alternatively, combiners that compromise linearity in order to significantly improve the transmitter's power efficiency may be used, such as the Chireix combiner [12] that is also shown in Fig. 1 as part of the LINC amplifier. According to [12], the envelope $r_{o}(t)$ of the recombined signal $s_{o}(t)$ can in this case be written as:

$$
r_{o}(t)=G_{c} r_{\max } \cos (\theta(t)-\lambda),
$$

where

$$
G_{c}=\frac{2 z_{c} G \cos (\lambda)}{z_{c}^{2}+2 \cos ^{2}(\lambda)}
$$

is the combination method's gain, which is a constant term that depends on the circuit's electrical parameters: $G$ is the amplifiers' gain, $z_{c}=Z_{c} / Z_{o}$ represents the normalized characteristic impedance of the combiner's quarter-wave transmission lines, $Z_{o}$ is the output load's impedance, $B$ is the stubs' susceptance, and $\lambda=\arctan \left(B \cdot Z_{0}\right)$ is the stubs' electrical length. Despite the clear differences between a Chireix combiner without stubs and a passive combiner ( [9] provides a detailed study on the subject), equation (6) is also valid for a passive combiner with $G_{c}=G$ and $\lambda=0$. Moreover, $r_{o}(t)$ given by (6) is an amplified and phase-shifted version of (2), which means that $\lambda \neq 0$ yields a nonlinear amplification gain for the Chireix combiner.

Due to the flexibility offered by digital signal processing, LINC separation is usually performed in the discrete time domain [9], [22], [23], along with, the bandwidth limitation performed by pulse shaping (typically using a root raised cosine (RRC) filter [24]) on the modulated signal before the LINC amplification stage. Converting equations (1)-(6) to the digital domain is a trivial operation, and from now on any reference to them will be alluding to their digital equivalent representation.

Despite the LINC's technique potential efficiency gains, its implementation faces challenges that result from the signal separation and recombination procedures, and from the imbalances between the HPAs, which are examined in the following.

\section{B. Broad Spectra LINC Signal Components}

The high power efficiency of the LINC transmitter arises from employing highly efficient, nonlinear HPAs to amplify two constant-envelope signals $s_{1}$ and $s_{2}$. The signal separation performed by the LINC structure, by either computing (3) or (4), can be regarded as phase modulating the LINC

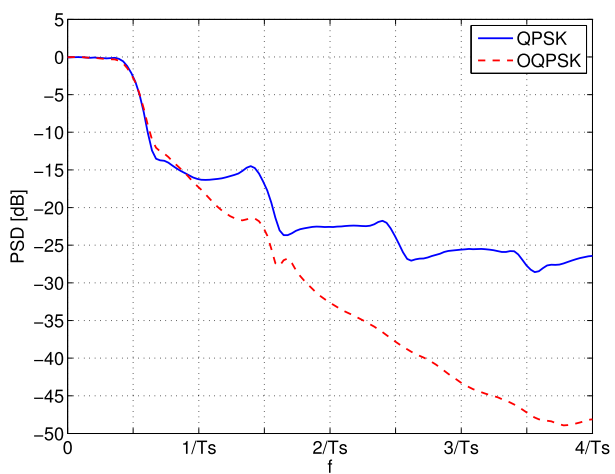

Fig. 2. LINC component's PSD for bandlimited QPSK and OQPSK signals, respectively, where $T_{S}$ denotes the symbol period. The employed RRC filter has a $25 \%$ roll-off factor.

components with the information stored within the original signal, as it is more easily perceived from (3). Accordingly, the LINC components have a much larger bandwidth than the input bandlimited signal $s_{n}$, and their spectrum broadens as the envelope fluctuations increases [9].

In order to keep the constant-envelope characteristics of $s_{1}$ and $s_{2}$, the nonlinear HPAs are required to accommodate the bandwidth of the LINC components. The same care should be taken while designing the digital-to-analog converters (DACs)' reconstruction filter; however, the order of these filters can be significantly reduced by using a higher oversampling rate [24]. Regarding this, Fig. 2 depicts the spectrum of the LINC components that result from a QPSK and an OQPSK signal, respectively; $s_{1}$ and $s_{2}$ have an identical power spectrum so, without loss of generalization, only the spectrum of one of these components is shown. While a high oversampling factor $L$, with $L \geq 16$, is critical for a feasible transmission system using the QPSK modulation scheme [25], this factor is much less restrictive when it is used an OQPSK digital modulator (e.g., $L=8$ ) [20], due to the much lower bandwidth requirements for the LINC signal components computed from the OQPSK signal, as it is easily perceived from Fig. 2. This is consistent with the idea that an envelope with lower fluctuations produces LINC components with a narrower spectrum.

Another problem to take care relates to the gain and phase imbalances between the nonlinear HPAs that amplify the LINC components. While theoretically, the signal reconstruction performed by a LINC linear combiner assumes each the nonlinear HPAs have the same amplification gain [8], with the envelope of LINC's output signal being given by $G_{c} r(t)$, this may not be the case in practice, and it may lead to a significant performance degradation [9], [19]. According to [25] and [19] the transmitted signal's spectrum experiences an increase of out-of-band radiation as the HPAs become more unbalanced; however the reduction of the dynamic range of input signal's envelope increases the system's robustness against to imbalances. Additionally, the LINC transmitter is more sensitive to phase imbalances [9], [10], as it is expected, since the constant-envelope LINC signal components $s_{1}$ and $s_{2}$ keep the signal's information in their phases.

For LINC transmitters that employ the Chireix combiner, there is the additional spectral spreading problem associated 
with its nonlinear amplification gain, expressed in signals' output envelope (6) by a phase-shift $\lambda$. Since a reduced range of the decomposition angle $\theta$ yields a combiner's gain with a smaller range and a more homogeneous distribution (see (2) and (6)), it is reasonable to expect that reducing the envelope fluctuations of $s_{n}$ will mitigate the undesirable outof-band radiation levels, as long as this is performed before the pulse shaping operation.

\section{The Power Combiner's Role on the Transmitter's Efficiency}

The main feature of the LINC technique lies on the use of highly efficient nonlinear HPAs to amplify the transmitted signal. However, it is the output signal's remaining power that dictates the efficiency of the amplifying process, giving to the LINC combiner a key role on the transmitter scheme [9].

As in (6), it is possible to use a single expression to describe the power efficiency of a LINC system employing either a passive or a Chireix combiner. According to [12], the combiners' instantaneous efficiency can be written as

$$
\eta_{\text {comb }}=K\left(\lambda, z_{c}\right) \cos ^{2}(\theta-\lambda),
$$

where, for the Chireix combiner,

$$
K\left(\lambda, z_{c}\right)=\frac{8 z_{c}^{2} \cos ^{2}(\lambda)}{\left(z_{c}^{2}+2 \cos ^{2}(\lambda)\right)^{2}}
$$

is a constant that depends solely on the circuit's electrical parameters, while for the matched combiner, the efficiency is given by (8) considering $K=1$, and $\lambda=0$.

In both cases, the transmitter's designer wishes to maximize the combiner's average efficiency $\bar{\eta}_{c o m b}=E\left[\eta_{\text {comb }}\right]$, which depends mainly on the probability density function (PDF) of the LINC's decomposition angle $\theta$, expressed by $p_{\theta}(\theta)$, and with the maximum of $\bar{\eta}_{c o m b}$ being given by

$$
\max \left\{\bar{\eta}_{c o m b}\right\}=\max \left\{\int_{0}^{\pi / 2} K \cos ^{2}(\theta-\lambda) p_{\theta}(\theta) \mathrm{d} \theta\right\} .
$$

1) Passive Combiner (PC): Setting $K=1$, and $\lambda=0$ on (8), it results

$$
\bar{\eta}_{c o m b, P C}=\int_{0}^{\pi / 2} \cos ^{2}(\theta) p_{\theta}(\theta) \mathrm{d} \theta,
$$

showing that the combiner's efficiency relies only on $\theta$ and its PDF, $p_{\theta}(\theta)$. Therefore, to improve the passive combiner's power efficiency it is required to adjust $p_{\theta}(\theta)$ (that is highly dependent on the envelope excursion of bandlimited signal $s_{n}$ ) to the instantaneous efficiency curve $\eta_{c o m b}=\cos ^{2}(\theta)$. In fact, by rewriting (10) using (2) and employing the equivalent PDF of $r(t)$, the average efficiency for the matched combiner becomes

$$
\bar{\eta}_{c o m b, P C}=\int_{0}^{r_{\max }} \frac{r^{2}}{r_{\max }^{2}} p_{r}(r) \mathrm{d} r=\frac{\bar{r}^{2}}{r_{\max }^{2}}=\frac{1}{\mathrm{PAPR}},
$$

which shows that the passive combiner's efficiency is directly related to the incoming signal's PAPR, and thus can be improved by reducing the envelope fluctuations of $s_{n}$. As a consequence, the range of $\theta$ is reduced, and thus moved to lower values, therefore maximizing the efficiency $\cos ^{2}(\theta)$.
2) Chireix Combiner $(C C)$ : In this case, the combiner's efficiency is related not only to the envelope of $s_{n}$ (via the decomposition angle) but also to the circuit's electrical parameters, that should be tuned to maximize $\eta_{\text {comb }}$. Given that in (8), among the circuit parameters, the cosine term depends only on the stubs' electrical length $\lambda$, the maximum efficiency can be achieved by finding first the optimal $z_{c}$ as a function of $\lambda$ that maximizes (9), i.e. that yields $K=1$, which is easily shown to be [12]

$$
z_{\text {opt }}=\sqrt{2} \cos (\lambda) \text {. }
$$

Moreover, unlike in the passive combiner case, there is a degree of freedom associated with the stubs' electrical length $\lambda$, which can be used to shift the instantaneous efficiency curve towards the more likely values of $\theta$ [12]. Having knowledge of $p_{\theta}(\theta)$, which can easily be estimated by simulation, the $\lambda$ value that maximizes (10) (here referred to as $\lambda_{\text {opt }}$ ) can be determined considering $K=1$ and differentiating (10) with respect to $\lambda$, followed by finding the resulting expression's zero value, as follows:

$$
\begin{aligned}
& \frac{d}{d_{\lambda}} \int_{0}^{\frac{\pi}{2}} \cos ^{2}(\theta-\lambda) p_{\theta}(\theta) d \theta \\
& \quad=0 \Leftrightarrow \int_{0}^{\frac{\pi}{2}} \sin (2(\theta-\lambda)) p_{\theta}(\theta) d \theta=0 .
\end{aligned}
$$

The value of $z_{\text {opt }}$ that assures that $K=1$ is then obtained by computing (13) using $\lambda_{\text {opt }}$.

As in the case of the passive combiner, the efficiency of a LINC system employing a Chireix combiner greatly benefits from reducing the envelope and PAPR of the signal to amplify because this reduces considerably the range of $\theta$, wherefore minimising the difference $\theta-\lambda_{\text {opt }}$, and thus making the term $\cos ^{2}(\theta-\lambda)$ approach to 1 .

As for the decomposition angle $\theta$, the analysis previously made regarding the matched combiner, i.e. a reduced $\theta$ range improves the combiner's efficiency, is still valid when a Chireix combiner is employed.

\section{Ring-Type Magnitude Modulation}

It is known that OQPSK signals can be used to represent any M-ary constellation [13], [26], and the transmission of several OQPSK signals in parallel using multi-layer massive MIMO transmitter schemes for high-order constellations has been recently proposed in [15], [16] to meet 5G's demanding throughput and spectral efficiency. This combined with the use of LINC techniques may leverage the development of transmitters with high power efficiency. However, the use of Nyquist pulse shaping to limit the bandwidth of the signals making it close to the minimum Nyquist band, introduces undesirably excursions on the signals envelope, which even for OQPSK may produce a signal with an envelope having a high dynamic range that considerable limits the efficiency of LINC's combiner and overall LINC's efficiency, while requiring for the use of amplifiers with large bandwidth.

Magnitude modulation techniques [17], [18], [27], can provide a suitable solution. MM was originally developed to limit the maximum excursion of bandlimited SC signals of generic $\mathrm{M}$-ary constellations (thus requiring for linear amplification) in 
order to reduce input back-off requirements of linear HPAs to improve SC transmitter's energy efficiency; moreover, since MM operates before pulse shaping it does not affect signal bandwidth. However, just limiting the maximum excursions is not enough to allow an efficient LINC implementation because, as seen previously, improving LINC's efficiency is mainly dependent on reducing the range of the LINC decomposition angle, $\theta$, directly related to the excursion range of the signal's envelope, which can only be effectively reduced by controlling both its maximum and minimum values.

In order to enable efficient LINC amplification of OQPSK signals, in this section we present a new Ring-type Magnitude Modulation (RMM) technique [20] for bandwidth limited OQPSK signals which simultaneously controls the envelope's minimum and maximum excursions. The new RMM method uses two different coefficients for the OQPSK symbols' in-phase and quadrature components that are computed $a$ priori and stored in look-up tables (LUTs).

\section{A. MM Principle}

Let $x_{n}$ denote a sequence of modulated symbols from a given constellation (e.g. OQPSK, M-ary QAM, etc.) and $s_{n}$ the corresponding pulse shaped sequence by employing Nyquist pulse shaping using a filter with impulse response $h_{n}$. Despite the differences between existing MM methods, each scheme inherently applies the following steps:

- For each new generated modulated symbol $\hat{x}_{n}$, predict the output response of the pulse shaping filter, by considering neighbouring symbols of $\hat{x}_{n}$ to some time extent that depends on the filter length;

- Detect the peaks of the predicted response above a specified threshold on that interval and calculate the corresponding scaling factor(s) to apply to $\hat{x}_{n}$;

- Multiplying the symbol $x_{n}$ by its MM coefficient $m_{n}$.

By adjusting each symbol before the bandwidth limiting pulse shaping operation, the typical magnitude modulated signal $s_{n}$ is described by:

$$
s[n]=\left[\sum_{k} m[k] x[k] \delta[n-k L]\right] * h[n],
$$

where the MM coefficients $m_{n}$ are computed to keep $s_{n}$ 's envelope below the amplitude threshold $A$, i.e.:

$$
|s[n]| \leq A .
$$

While enforcing condition (16), the maximum excursion of the signal's envelope is considerably reduced and, consequently, the signal's PAPR; note that, although a net PAPR reduction gain is obtained with conventional MM techniques, these do not directly control the PAPR since the signal's average power is also reduced with the MM procedure. This reduction on the signal's PAPR is enough to significantly upgrade a transmitter that employs a linear HPA (in terms of performance and power efficiency). However, that is not the case for a LINC transmitter due to the constraints associated to the signal separation and combination discussed in section II. To assess these, the LUT-based MM method [17] was applied to an OQPSK signal with power $\sigma^{2}$, and combined with the
LINC, considering a transmitter scheme similar to the one used in [25] for the quadrature phase shift keying (QPSK) case. Although this conventional MM scheme reduces the OQPSK signal's PAPR (over $2 \mathrm{~dB}$ reduction at a probability of $10^{-4}$ for the stringent limit $A / \sigma=1$ ), the bandwidth of the LINC components is only slightly reduced, occupying nearly three times the bandwidth of $s_{n}$.

The undesirable characteristics of the LINC components when using conventional MM happens because enforcing only an upper bound on the envelope excursions also results in scaling down the lower valued samples, which does not produce neither a smaller envelope range nor a smaller decomposition angle $\theta$ range, as required. Therefore, to guarantee both a PAPR and envelope dynamic range reduction, while also avoiding envelope's zero crossings, it is necessary also to add a lower amplitude threshold. The new proposed ringtype magnitude modulation algorithm incorporates this new restriction looking for a different solution from traditional MM methods (e.g. [17], [28]).

\section{B. Ring-Type Magnitude Modulation}

Considering the LINC requirements discussed so far and the need for spectral efficient communications systems, the RMM technique was developed for the OQPSK modulation scheme [20]. Since the peak values of the OQPSK signals' in-phase and quadrature components do not occur at the same time (due to their half symbol-period time offset), it is possible to further confine its already low fluctuating envelope to an upper and a lower boundary without severely distorting the transmitted signal. Accordingly, the proposed RMM method exploits the referred time offset by using two scaling coefficients (i.e. one for each symbol component), which provides a finer control of the envelope excursions at the expense of adding some phase modulation.

Considering all this, (15) is rewritten as:

$$
\begin{aligned}
s[n]= & {\left[\sum_{k} m_{I}[k] x_{I}[k] \delta[n-k L]\right] * h[n] } \\
& +\left[\sum_{k} m_{Q}[k] x_{Q}[k] \delta\left[n-k L-\frac{L}{2}\right]\right] * h[n],
\end{aligned}
$$

where $m_{I}$ and $m_{Q}$ refer respectively to the in-phase and quadrature components' RMM coefficients. Furthermore, each RMM signal is designed in order to satisfy the following condition:

$$
A_{l} \leq|s[n]| \leq A_{u}
$$

where $A_{l}$ and $A_{u}$ represent, respectively, the RMM's lower and upper amplitude boundaries.

Unlike other MM methods (e.g. multistage polyphase magnitude modulation (MPMM) [18]), the task of developing a real-time method is not straightforward due to the number of RMM parameters (two amplitude boundaries and two coefficients) aiming at obtaining a magnitude modulated signal having an envelope with very low dynamic range and without zero crossings, which is why the RMM coefficients are computed a priori and stored in LUTs, as in classical MM 